\title{
Improved detection limits using a hand-held optical imager with coregistration capabilities
}

\author{
Sarah J. Erickson, Sergio L. Martinez, Jean Gonzalez, Lizeth Caldera, and Anuradha \\ Godavarty* \\ Department of Biomedical Engineering, Florida International University, 10555 West Flagler Street EC2610; Miami, \\ FL 33174, USA \\ *godavart@fiu.edu
}

\begin{abstract}
Optical imaging is emerging as a non-invasive and non-ionizing method for breast cancer diagnosis. A hand-held optical imager has been developed with coregistration facilities towards flexible imaging of different tissue volumes and curvatures in near real-time. Herein, fluorescenceenhanced optical imaging experiments are performed to demonstrate deeper target detection under perfect and imperfect (100:1) uptake conditions in (liquid) tissue phantoms and in vitro. Upon summation of multiple scans (fluorescence intensity images), fluorescent targets are detected at greater depths than from single scan alone.
\end{abstract}

(C)2010 Optical Society of America

OCIS codes: (170.3880) Medical and Biological Imaging; (110.2970) Image Detection Systems; (110.0113) Imaging through turbid media; (110.3080) Infrared imaging; (110.6955) (300.2530) Fluorescence, laser-induced

\section{References and links}

1. S. J. Erickson, and A. Godavarty, "Hand-held based near-infrared optical imaging devices: a review," Med. Eng. Phys. 31(5), 495-509 (2009).

2. A. E. Cerussi, A. J. Berger, F. Bevilacqua, N. Shah, D. Jakubowski, J. Butler, R. F. Holcombe, and B. J. Tromberg, "Sources of absorption and scattering contrast for near-infrared optical mammography," Acad. Radiol. 8(3), 211-218 (2001).

3. N. Shah, A. E. Cerussi, D. Jakubowski, D. Hsiang, J. Butler, and B. J. Tromberg, "Spatial variations in optical and physiological properties of healthy breast tissue," J. Biomed. Opt. 9(3), 534-540 (2004).

4. A. Cerussi, N. Shah, D. Hsiang, A. Durkin, J. Butler, and B. J. Tromberg, "In vivo absorption, scattering, and physiologic properties of 58 malignant breast tumors determined by broadband diffuse optical spectroscopy," J. Biomed. Opt. 11(4), 044005 (2006).

5. B. Chance, S. Nioka, J. Zhang, E. F. Conant, E. Hwang, S. Briest, S. G. Orel, M. D. Schnall, and B. J. Czerniecki, "Breast cancer detection based on incremental biochemical and physiological properties of breast cancers: a six-year, two-site study," Acad. Radiol. 12(8), 925-933 (2005).

6. B. Chance, Z. Zhao, S. Wen, and Y. Chen, "Simple ac circuit for breast cancer detection and object detection," Rev. Sci. Instrum. 77(6), 064301 (2006).

7. J. Ge, B. Zhu, S. Regalado, and A. Godavarty, "Three-dimensional fluorescence-enhanced optical tomography using a hand-held probe based imaging system," Med. Phys. 35(7), 3354-3363 (2008).

8. J. Ge, S. J. Erickson, and A. Godavarty, "Fluorescence tomographic imaging using a handheld-probe-based optical imager: extensive phantom studies," Appl. Opt. 48(33), 6408-6416 (2009).

9. S. J. Erickson, J. Ge, A. Sanchez, and A. Godavarty, "Two-dimensional fast surface imaging using a handheld optical device: In vitro and in vivo fluorescence studies," Transl Oncol 3(1), 16-22 (2010).

10. S. Regalado, S. J. Erickson, B. Zhu, J. Ge, and A. Godavarty, "Automated coregistered imaging using a handheld probe-based optical imager," Rev. Sci. Instrum. 81(2), 023702 (2010).

11. A. Godavarty, C. Zhang, M. J. Eppstein, and E. M. Sevick-Muraca, "Fluorescence-enhanced optical imaging of large phantoms using single and simultaneous dual point illumination geometries," Med. Phys. 31(2), 183-190 (2004).

12. A. Corlu, R. Choe, T. Durduran, M. A. Rosen, M. Schweiger, S. R. Arridge, M. D. Schnall, and A. G. Yodh, "Three-dimensional in vivo fluorescence diffuse optical tomography of breast cancer in humans," Opt. Express 15(11), 6696-6716 (2007).

\section{Introduction}

Optical imaging is a growing area of research, with efforts to translate the technology to the clinic and aid in early-stage breast cancer detection, diagnosis, and/or prognosis of the disease. Optical imaging systems are developed by various research groups with differences in 
their imaging technique (continuous-wave, frequency-domain, or time-domain), and sourcedetector configuration (single or multiple sources/detectors that are either for point/area imaging). While most of these systems are bulky and yet capable of three-dimensional (3D) tomographic imaging of large tissue volumes, there are a whole class of hand-held optical imaging systems that are portable and capable of two-dimensional (2D) spectroscopic imaging [1-6].

In the past five years, our Optical Imaging Laboratory has been focused in developing a hand-held (portable) optical imaging system that is capable of 3D tomographic imaging beyond the 2D spectroscopic imaging capabilities (available to date) [7]. The recently developed hand-held optical imager is unique in its following features: (i) a probe head to image a large surface area simultaneously; (ii) a simultaneous illumination and detection geometry that enhances the overall imaging time; (iii) a flexible probe head to contour to any tissue curvature and volume; and (iv) a novel coregistration approach to acquire the positional information using a motion tracker (towards 3D tomography studies). The feasibility of 2D target detection(s) and 3D tomographic analysis using this hand-held optical imager has been demonstrated from tissue phantom and in-vitro fluorescence imaging studies [7-9]. During these studies, a single scan in the region of interest was acquired to detect and recover the embedded target(s) tomographically (using manually coregistered optical images). However, it was observed that as the target depth was increased and/or its volume decreased, the target was not detectable from 2D images, nor was it recovered from 3D tomography studies. An alternate imaging approach of using multiple-scans from various locations of the tissue surface was developed (using automatically coregistered optical images) and its feasibility to detect deeper targets was assessed [10].

In the current work, the recently developed multi-scan imaging approach is extensively assessed to determine the target detection limits of our hand-held optical imager. Studies were performed using the liquid tissue phantoms in order to determine the extent of improvement upon the previous studies using single-scan. Studies were also extended to in vitro tissue models in order to assess the performance of the device in a non-uniform (or heterogeneous) scattering background, in order to better mimic human tissue.

\section{Materials and methods}

\subsection{Instrumentation}

The instrumentation for the hand-held optical imaging system as shown in Fig. 1 is composed of a laser diode source $(785 \mathrm{~nm}, 530 \mathrm{~mW}$ ) and intensified charge-coupled device (ICCD) detector ( $-24 \mathrm{~V} \mathrm{DC}$ detector power), which are connected to the probe face via optical fibers. The $4 \times 9 \mathrm{~cm}^{2}$ hand-held probe head contains 6 illumination points (shown as large red dots in Fig. 1) and 165 detection points (spaced $0.5 \mathrm{~cm}$ apart) which illuminate and collect the signal simultaneously for rapid data acquisition. A collimator-diffuser package splits the NIR light from the laser diode into 6 simultaneous sources. The laser output from each of these 6 sources at the probe end onto the tissue geometry is only $2 \mathrm{~mW}$ or less at each illumination point (due to $>90 \%$ intensity losses from the collimator-diffuser package). The imaging system is capable of operating in both the continuous wave $(\mathrm{CW})$ and frequency domain mode. The CW mode is used here as a fast measurement technique (near real-time) to enable collection of multiple scans without greatly increasing the imaging time. During imaging studies, the integration time of the ICCD camera is kept constant $(0.2 \mathrm{sec})$ and only the gain settings at the intensifier end is varied. Further details of the instrumentation have been described elsewhere [7]. 


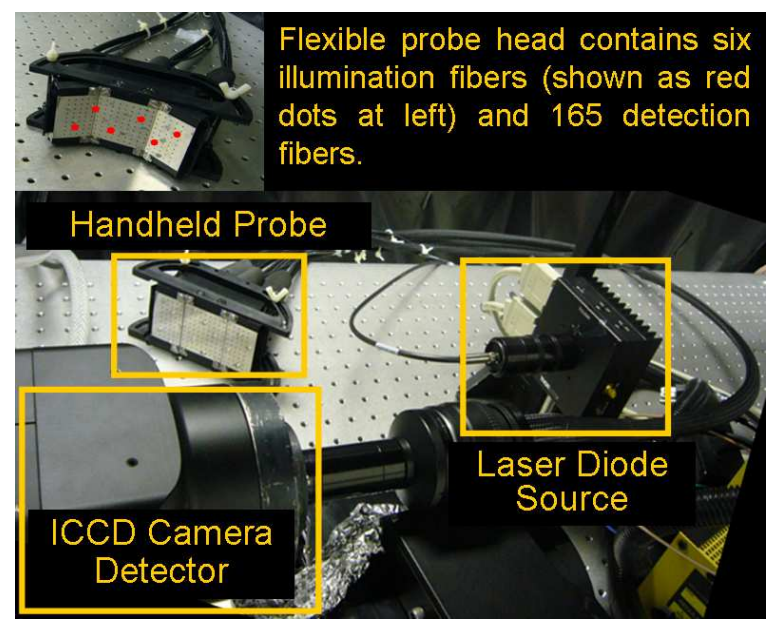

Fig. 1. Instrumentation for the hand-held probe based optical imaging system.

\subsection{Coregistered imaging process}

Image coregistration onto the 3D tissue geometry being scanned is necessary in order to perform 3D tomography studies. This is because the location of the image must be known with respect to the geometry of the phantom (or tissue) being imaged. Coregistered imaging involves tracking the position and orientation of the probe in real time and combining the positional information with the optical measurements to accurately position the image on the discretized phantom or tissue geometry. This is carried out as a three-step process (illustrated in Fig. 2) using MATLAB/LabVIEW software developed in house [10]. An acoustic tracker is implemented on the probe head to enable real-time tracking of the 3D position and orientation of the probe (in six degrees of freedom) with respect to the phantom surface (Step \#1). The positional information is used to accurately position the $2 \mathrm{D}$ image of fluorescence intensity at the corresponding location on the discretized phantom geometry. The positional information is fused with the optical measurement data collected from the hand-held device (Step \#2) to generate a 3D coregistered image of the phantom with the fluorescence intensity (or any other optical measurement) data at the probe's location (Step \#3). The entire coregistration process is automated such that it takes $\sim 35$ seconds to acquire a single coregistered image or scan (i.e. carry out all the 3 steps described above). The actual time to acquire an optical image at a single location of the probe is $\sim 1 \mathrm{sec}$ (i.e. $0.2 \mathrm{sec}$ detector integration time $\times 5$ repetitions for each CW image), and the remaining time $(\sim 34 \mathrm{sec})$ is towards the coregistration process. The coregistration process is repeated at each probe location, during a multi-scan imaging technique (i.e. optical images are acquired from multiple locations of the probe on the tissue surface).

\subsection{Validation of coregistered imaging in phantoms}

In order to quantitatively determine the accuracy of the tracked location in comparison to the true location of the probe on a cubical tissue phantom, the probe was placed at five different positions of known $[\mathrm{x}, \mathrm{y}, \mathrm{z}]$ coordinates and the tracking position was recorded (5 repetitions at each location). The average and standard deviation of the tracked location, and its total distance-off from the true location at each position number were measured. The average total distance off is $\sim 0.19 \mathrm{~cm}$. For these measurements, the probe was held in place with a lab jack and the error in measurements is primarily due to error in the tracking system and possibly some movement of the phantom during positioning of the probe. Currently work is carried out to improve the accuracy of the tracking system. 


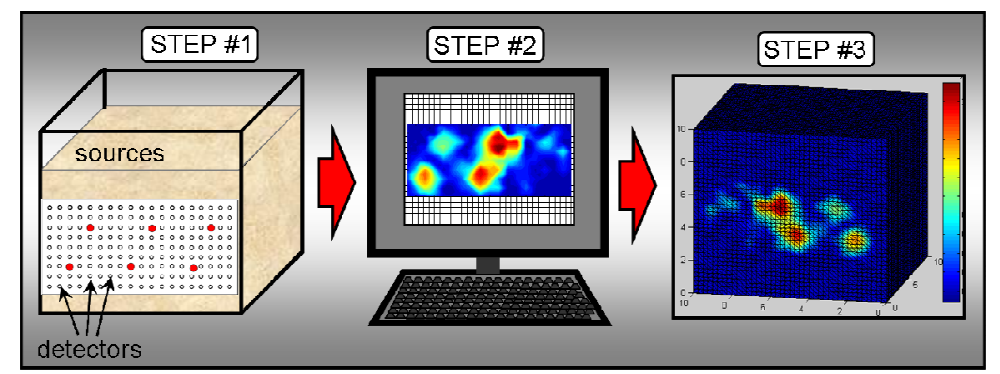

Fig. 2. Three-step coregistered imaging process. In Step \#1, the source and detector locations are tracked in real-time with respect to the phantom. In Step \#2, a raw image (of optical measurement) is collected and used to generate a 2D surface contour plot of the corresponding (here fluorescence intensity) data. In Step \#3, the positional information is used to accurately coregister the image to the probe's location on the discretized phantom mesh.

\subsection{Experimental studies}

Experimental studies were performed initially using the simple case of the uniform liquid tissue phantom and were then extended to more realistic in vitro models. Phantom experiments were performed using tissue phantoms (as in the previous studies $[7,8]$ ) composed of $1 \%$ Liposyn $(800 \mathrm{~mL})$ in a $10 \times 10 \times 10 \mathrm{~cm}^{3}$ acrylic cube. Acrylic sphere targets of sizes $0.23-0.45 \mathrm{~cm}^{3}$ filled with $1 \mu \mathrm{M}$ indocyanine green (ICG) were placed at different depths $(2.5-4.0 \mathrm{~cm})$ from the imaging surface to represent a tumor. Experiments were performed increasing the depth by $0.5 \mathrm{~cm}$ until the target was no longer detected. The experimental set-up is shown in Fig. 3. The probe was placed in full contact with the phantom surface and multiple scans (2D coregistered images) were collected using the method described in section 2.5 below.
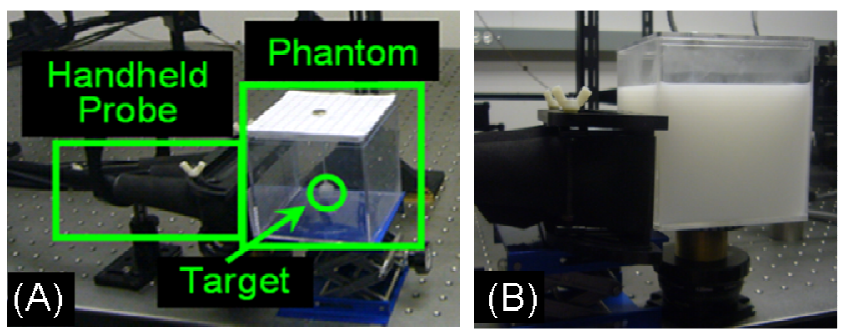

Fig. 3. Experimental set-up for phantom studies. (A) A spherical target filled with $1 \mu \mathrm{M}$ indocyanine green is enclosed within the cubical phantom to represent a tumor. (B) The phantom is composed of a $1 \%$ Liposyn solution to mimic the optical properties of human breast tissue.

To represent the heterogeneous nature of human tissue, studies were also performed in vitro using minced chicken breast $(480 \mathrm{~mL})$ combined with $1 \%$ Liposyn $(260 \mathrm{~mL})$ in a $10 \times$ $10 \times 10 \mathrm{~cm}^{3}$ acrylic cube in order to introduce a background of non-uniform scattering. The $0.45 \mathrm{~cm}^{3}$ fluorescent target was placed at different depths between 2.0 and $4.0 \mathrm{~cm}$, until the target was no longer detected. The experiments were performed for the perfect uptake case with tumor-to-background ratio (T:B) of 1:0 and imperfect uptake case with T:B of 100:1. Table 1 summarizes the different experimental studies performed in which a target was detected. For each image collected, a subtraction-based post-processing technique was used to eliminate excitation light leakage [7].

\subsection{Multiple-scan technique}

The multiple scan technique involves collecting a series of scans at different probe positions and coregistering each image to its appropriate location. A scan is defined as a 2D surface contour plot of fluorescence intensity data collected from a CW image (mean of 5 repeated 
images at the same location) using the ICCD based detection system. Each image is immediately coregistered to the discretized phantom mesh. By scanning at multiple locations, the weak fluorescence signal from the target (that appears at the same location on the tissue geometry during each scan) can possibly dominate the strong excitation light leakage (that tends to appear at different locations on the tissue geometry during each scan).

\begin{tabular}{|c|c|c|c|c|}
\hline $\begin{array}{l}\text { Experimental } \\
\text { Case }\end{array}$ & Experiment \# & $\begin{array}{c}\text { Target Volume } \\
\left(\mathrm{cm}^{3}\right)\end{array}$ & $\begin{array}{l}\text { Target Depth } \\
(\mathrm{cm})\end{array}$ & $\mathrm{T}: \mathrm{B}$ \\
\hline Tissue & 1 & 0.45 & 3.0 & $1: 0$ \\
\hline \multirow[t]{9}{*}{ Phantom } & 2 & 0.45 & 3.5 & 1:0 \\
\hline & 3 & 0.23 & 2.5 & $1: 0$ \\
\hline & 4 & 0.23 & 3.0 & $1: 0$ \\
\hline & 5 & 0.23 & 3.5 & $1: 0$ \\
\hline & 6 & 0.45 & 2.5 & 100:1 \\
\hline & 7 & 0.45 & 3.0 & $100: 1$ \\
\hline & 8 & 0.23 & 2.0 & 100:1 \\
\hline & 9 & 0.23 & 2.5 & 100:1 \\
\hline & 10 & 0.23 & 3.0 & 100:1 \\
\hline \multirow[t]{5}{*}{ In Vitro } & 11 & 0.45 & 2.5 & $1: 0$ \\
\hline & 12 & 0.45 & 3.0 & $1: 0$ \\
\hline & 13 & 0.45 & 3.5 & $1: 0$ \\
\hline & 14 & 0.45 & 2.0 & 100:1 \\
\hline & 15 & 0.45 & 2.5 & $100: 1$ \\
\hline
\end{tabular}

Different combinations of image positions were used to determine the optimal multi-scan method. Initially 4-5 scans were collected moving the probe $0.5 \mathrm{~cm}$ in the vertical direction between each scan. For the same experimental case, 9 scans were collected moving the probe in $0.25 \mathrm{~cm}$ increments in the vertical direction. Additionally, repeated scans were collected at each probe location. It was determined that collecting 2-3 repeated scans (i.e. $2-3 \times 5$ repeated images or 10-15 repeated images) at each probe location for $4-5$ positions $0.5 \mathrm{~cm}$ apart resulted in better target detectability with fewer artifacts than collecting single scans (i.e. $1 \times 5$ repeated images) at each probe location for 9 positions $0.25 \mathrm{~cm}$ apart. This can possibly be attributed to two reasons: (i) the inaccuracy (from instability) of the positional tracking system (described in section 2.3) has greater impact at smaller positional increments (e.g. $0.25 \mathrm{~cm}$ ), leading to increased artifacts; and (ii) the variability in the instrument's response is minimized by increasing the number of repeated scans (or images) at the same location. The number of repeated scans (or images) can be increased for the case where the probe location $0.25 \mathrm{~cm}$ apart. However, this further increases the overall imaging time and hence was not attempted. In the future, for an in vivo case, multiple images can be collected and summed from arbitrary probe positions and the number of scans can be optimized to minimize the overall imaging time and number of artifacts. The position will be known since the image is immediately coregistered at its location.

\section{Results}

Figure 4 shows four single images (2D contour plots of fluorescence intensity) for experimental case \# 12 where a $0.45 \mathrm{~cm}^{3}$ fluorescent target was placed $3.0 \mathrm{~cm}$ deep in vitro under perfect uptake condition $(\mathrm{T}: \mathrm{B}=1: 0)$. Multiple scans were collected as described in section 2.5. In each image, the true target location is indicated by a black open circle. The images show that the target is not detected in a single scan, and only random distributions of artifacts (high intensity signals that appear in the image which do not originate from the target) are visible. Upon summation of eight scans, the fluorescent signal is detected at the target location (Fig. 5). This can be attributed to the random distribution of artifacts that appear in different locations for each single scan, while the signal from the target remains in

\#129379 - \$15.00 USD

(C) 2010 OSA 
the same location relative to its coregistered position on the phantom. Upon summation of the coregistered images, the random signals from the artifacts tend to diminish compared to the consistent signal from the target, which tends to intensify.

Figure 6 shows the result for summated multi-scan images for experimental cases 5, 10, 13, and 15 (highlighted in Table 1). Case 5 represents a $0.23 \mathrm{~cm}^{3}$ target placed $3.5 \mathrm{~cm}$ deep in the tissue phantom under perfect uptake conditions $(\mathrm{T}: \mathrm{B}=1: 0)$. The target was detected close to the true location and no artifacts were present. Case 10 represents a $0.23 \mathrm{~cm}^{3}$ target placed $3.0 \mathrm{~cm}$ deep in the tissue phantom under imperfect uptake condition $(\mathrm{T}: \mathrm{B}=100: 1)$. The target was detected at the true location with some diffused signal around it. Case 13 represents a $0.45 \mathrm{~cm}^{3}$ target placed $3.5 \mathrm{~cm}$ deep in-vitro under perfect uptake conditions (T:B $\left.=1: 0\right)$. The target was detected at the true location with artifacts also visible far from the target location. These artifacts can be attributable to the heterogeneous nature of the in vitro phantom.

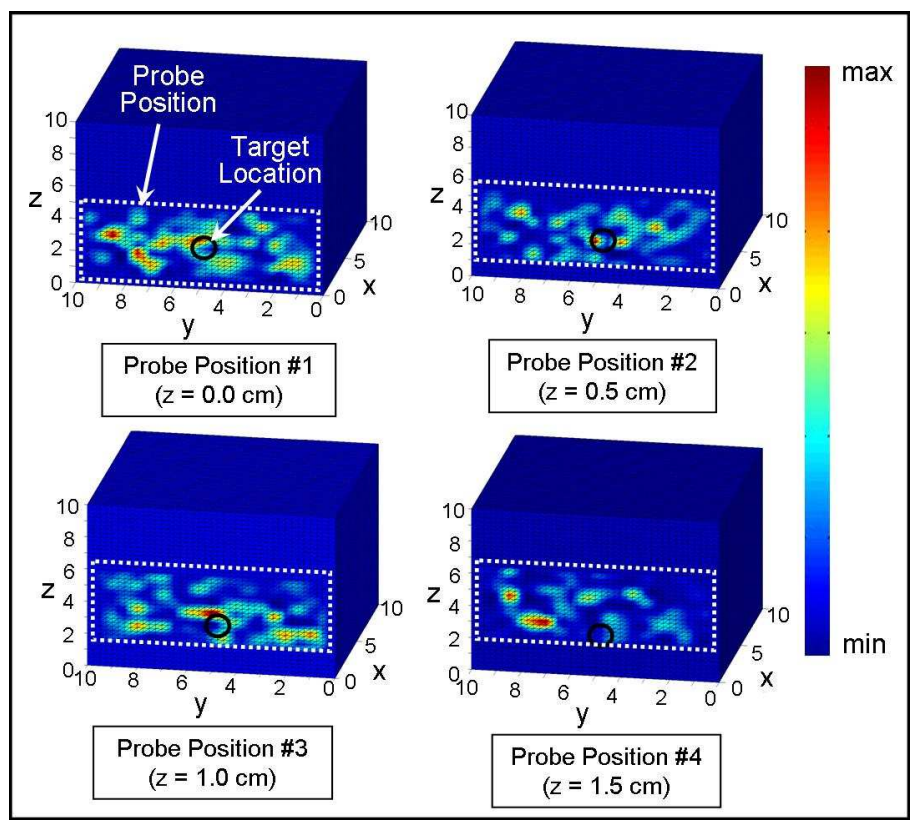

Fig. 4. Coregistered images from single scans (2D contour plots of fluorescence intensity data) at four probe positions for experimental case \#12 $\left(\mathrm{a} 0.45 \mathrm{~cm}^{3}\right.$ fluorescent target placed $3.0 \mathrm{~cm}$ deep, $\mathrm{x}$-dimension in-vitro phantom under $\mathrm{T}: \mathrm{B}=1: 0)$. In each image, the white dotted line represents the probe position with respect to the phantom and the black open circle represents the true target location. 


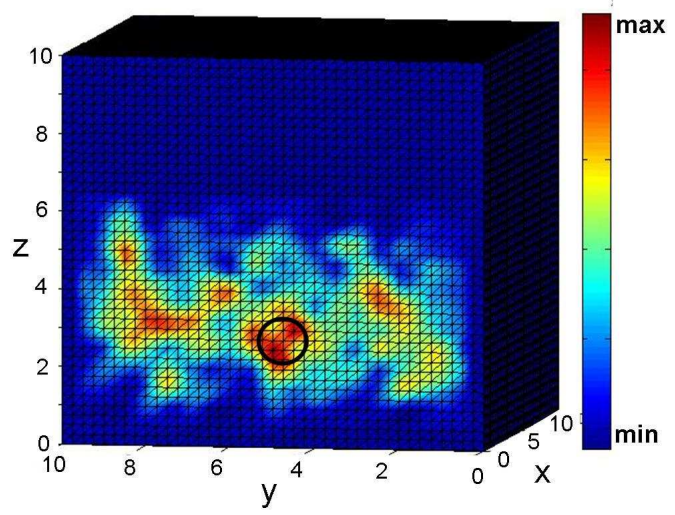

Fig. 5. Summated image of multiple scans shown in Fig. 4 (experimental case \#12). The summed image represents summation of 8 single scans, where 2 scans were collected at each of the 4 probe positions shown in Fig. 4. The black open circle represents the true target location.
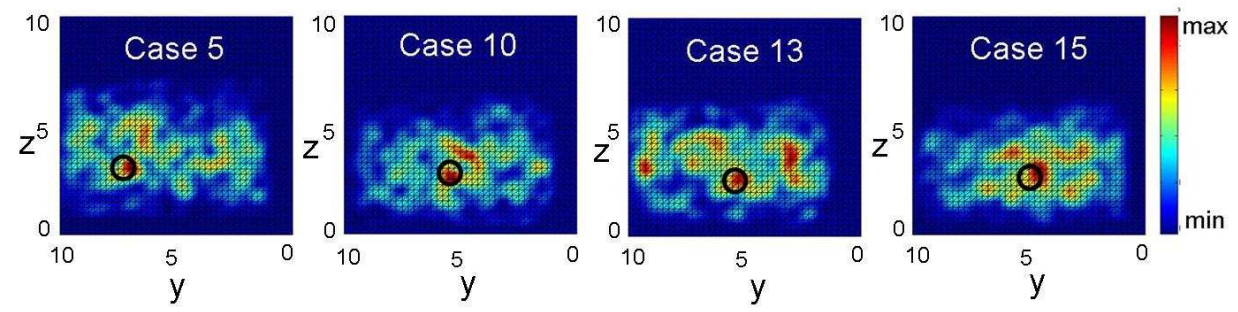

Fig. 6. Summed images of multiple coregistered scans from the four best experimental cases listed in Table 1. The black open circle indicates the true target location for each case.

Case 15 represents a $0.45 \mathrm{~cm}^{3}$ target placed $2.5 \mathrm{~cm}$ deep in-vitro under imperfect uptake conditions $(\mathrm{T}: \mathrm{B}=100: 1)$. The target was detected close to the true location along with minimal artifacts around it.

The initial experiments designed to detect deeper targets consisted of performing a single scan at 4-5 positions moving the probe $0.5 \mathrm{~cm}$ in the vertical (z) direction. For deeper targets $(>3.0 \mathrm{~cm}$ ), heterogeneous (in vitro) phantoms, and/or imperfect uptake cases, the target was not detected upon summation of four or five scans. However, it was observed that when multiple repeated scans were collected at the same location for each probe position, the targets were detectable. In other words, increasing the number of repeated scans is as important as increasing the number of scan locations towards deep target detection under heterogeneous and/or imperfect uptake conditions.

The results presented here demonstrate that deeper and smaller targets can be detected using the multiple-scan approach than using a single-scan alone. The impact of these results extend beyond target detection in $2 \mathrm{D}$ in that the information can be used as a priori information to aid $3 \mathrm{D}$ reconstruction using a single frequency-domain image (or scan) towards deeper target recovery (future work).

\section{Discussion}

The prime hypothesis for the current study is that a single scan (of a large area, $4 \times 9 \mathrm{~cm}^{2}$ ) can fail in detecting deeper targets during a near real-time optical imaging study. Upon scanning at multiple locations on the surface of a tissue geometry, the possibility to detect deeper targets and differentiating them from artifacts tends to improve upon summing all these 
multiple scans. The detection limits of our hand-held optical imager, the first of its kind that can scan large areas in near real-time, has been $2.5 \mathrm{~cm}$ for a $0.45 \mathrm{~cm}^{3}$ target under $\mathrm{T}: \mathrm{B}=1: 0$ from the past studies [8] involving a single scan from the region of interest on the tissue geometry. In the current study, it was clearly observed that upon summation of the intensity signals from multiple scans of the tissue surface, targets of smaller volume and as deep as 3.5 $\mathrm{cm}$ were detectable. However, artifacts start appearing when the target depth is increased, the target volume is decreased, and or heterogeneity of the background increased. The reasons for these artifacts are two-fold: (i) The uneven source strength distribution of the 6 simultaneous sources affects the ability to detect the target depending on the proximity of the target to the stronger sources. As multiple scans are collected the strong source may move away from the target resulting in a more diffused signal which produces a greater number of artifacts upon subtraction of the background. (ii) The positional information of the hand-held probe is not accurately coregistered due to the instability of the motion tracking device, which can lead to misalignment of the target signal in the multiple images. As a result, some signals which originated from the target can be mistaken as artifacts due to their improper location in the image. Currently, work is carried out to homogenize the source strength distribution by altering the instrumentation set-up at the source end, and also developing alternate motion tracking approaches that are stable and more accurate in comparison to the current device.

Researchers in the past have demonstrated detection of targets as deep as $5 \mathrm{~cm}$ using their hand-held optical devices [6]. However, the detection was typically a point location that was spectroscopically obtained from the tissue surface. In addition, the specificity of getting a negative result (not detecting any target) in the absence of the target (i.e. in the surrounding tissues) is not described. In other words, unlike the current hand-held optical imager that can produce 2D images over large areas in near real-time, the other hand-held imagers have a limited imaging area (mostly point-based imaging) and typically produce only spectroscopic measurements from a few sources/detectors.

The advantage of the current hand-held imager's optical data is that it can be applied towards 3D tomography studies, since the positional location of the optical data with respect to the $3 \mathrm{D}$ tissue geometry is coregistered during imaging studies. Hence, the implementation of the multi-scan imaging approach and using the summated images towards immediate 2D deep target detection and future 3D tomographic analysis is feasible. Although the present study is focused on fluorescence-enhanced optical imaging, the multi-scan summation and imaging approach is applicable for absorption-based diffuse optical imaging studies as well.

In the area of fluorescence tomographic imaging, Sevick's research group was one of the first groups to demonstrate 3D fluorescence optical tomography in clinically relevant sized tissue phantoms, using large bulky optical imaging instrumentation [11]. Fluorescent targets of $1 \mathrm{~cm}^{3}$ volume were recovered at depth up to $2.8 \mathrm{~cm}$ under perfect uptake conditions. The first in vivo 3D fluorescence optical tomography of breast cancer in human subjects was demonstrated by Corlu et. al. where tumors were recovered in vivo up to $2.0 \mathrm{~cm}$ deep in human breast tissue [12]. From our past 3D tomography studies using the hand-held imager on large tissue phantoms, $0.45 \mathrm{~cm}^{3}$ targets as deep as $2.5 \mathrm{~cm}$ were recovered in uniform tissue phantoms under perfect uptake conditions [8].

In a recent in vivo study using a simulated fluorescent target, a single scan using our handheld optical imager was able to detect a $0.23 \mathrm{~cm}^{3}$ target $\sim 2.5 \mathrm{~cm}$ deep in human breast tissue [9]. These past studies demonstrate the potential that upon using multiple scans and applying the summation approach, deeper and smaller targets can become detectable from the tissue surface. In addition, these summated images can also assist in tomographically recovering deeper targets beyond what is demonstrated to date in the area of fluorescence optical tomography. However, the developed hand-held optical imager is limited to reflectance based imaging, which tends to limit the recovery of the true target's depth during 3D tomography. Currently, research is carried out to develop alternate imaging approaches using the hand-held device such that trans-illumination measurements can also be acquired in an attempt to improve the target depth recovery (during 3D tomography). 


\section{Conclusion}

A hand-held optical imager has been developed with unique features of flexibility to contour to different tissue curvatures, ability to rapidly image a large area, and coregistration capabilities to enable 3D tomography. Herein we have demonstrated improved detection limits via application of a multiple-scan technique. The multi-scan imaging approach is facilitated by the use of fast $2 \mathrm{D}$ coregistered imaging in $\mathrm{CW}$ mode. Previous results using single-scan imaging showed that the greatest target depth detected was $2.5 \mathrm{~cm}$ and $1.5 \mathrm{~cm}$ for a $0.45 \mathrm{~cm}^{3}$ target in a (liquid) tissue phantom under perfect and imperfect uptake conditions, respectively. By using the multi-scan technique, this depth was improved to $3.5 \mathrm{~cm}$ for a smaller target $\left(0.23 \mathrm{~cm}^{3}\right)$ under the $\mathrm{T}: \mathrm{B}=1: 0$ condition. Under imperfect uptake conditions ( $\mathrm{T}: \mathrm{B}=100: 1)$, the detectable target depth was increased from $1.5 \mathrm{~cm}$ to $3.0 \mathrm{~cm}$. Since these results were promising, studies were extended to in vitro models, which better represent the non-uniformity of human tissue. From these in-vitro studies the target was detected at depths of $3.5 \mathrm{~cm}$ and $2.5 \mathrm{~cm}$ for perfect (1:0) and imperfect uptake (100:1) cases, respectively. These studies demonstrate that summation of multiple coregistered images can be used towards deeper target detection than is capable from single scans alone. In a clinical setting, this technique can be used to acquire multiple images quickly in order to detect the presence of a tumor, determine its 2D location within the tissue, and also perform 3D tomography studies (as a follow-up). Thus, the hand-held optical imager has potential for fast 2D imaging and 3D tomography in the clinical setting for breast cancer diagnosis.

\section{Acknowledgements}

The current work is supported by National Institutes of Health (R15CA119253), Dept of Defense (BC083282), and Coulter Foundation.

\#129379 - \$15.00 USD

(C) 2010 OSA
Received 1 Jun 2010; revised 10 Jul 2010; accepted 12 Jul 2010; published 15 Jul 2010

2 August 2010 / Vol. 1, No. 1/ BIOMEDICAL OPTICS EXPRESS 134 